\title{
QTRAJECTORIES: IMPROVING THE QUALITY OF OBJECT TRACKING USING SELF-ORGANIZING CAMERA NETWORKS
}

\author{
Uwe Jaenen $^{a}$, Udo Feuerhake ${ }^{b}$, Tobias Klinger ${ }^{c}$, Daniel Muhle $^{c}$, Joerg Haehner ${ }^{a}$, Monika Sester ${ }^{b}$, Christian Heipke $^{c}$ \\ Universitaet Augsburg - Lehrstuhl fuer Organic Computing ${ }^{a}$ \\ Leibniz Universitaet Hannover - Institut fuer Kartographie und Geoinformatik ${ }^{b}$ \\ Leibniz Universitaet Hannover - Institut fuer Photogrammetrie und GeoInformation ${ }^{c}$ \\ Appelstrasse 9a, 30167 Hannover, Germany \\ http://www.ikg.uni-hannover.de/index.php?id=696
}

KEY WORDS: Cooperation, Distributed, Automation, Organization, Pattern, Tracking, Networks, Performance

\begin{abstract}
:
Previous work in the research field of video surveillance intensively focused on separated aspects of object detection, data association, pattern recognition and system design. In contrast, we propose a holistic approach for object tracking in a self-organizing and distributed smart camera network. Each observation task is represented by a software-agent which improves the tracking performance by collaborative behavior. An object tracking agent detects persons in a video stream and associates them with a trajectory. The pattern recognition agent analyses these trajectories by detecting points of interest within the observation field. These are characterized by a non-deterministic behavior of the moving person. The trajectory points (enriched by the results of the pattern recognition agent) will be used by a configuration agent to align the cameras field of view. We show that this collaboration improves the performance of the observation system by increasing the amount of detected trajectory points by $22 \%$.
\end{abstract}

\section{INTRODUCTION}

In recent years video surveillance has become a ubiquitous safety procedure. Today's surveillance systems, which are in practical use, consist of optical sensors which stream image data in a central control room. The streams are analyzed manually by security staff or stored to save it as evidence. With an increasing number of them, the manual analysis of video streams and the configuration of the network becomes infeasible. The current research focuses on fully automated surveillance systems. This paper presents a holistic approach for distributed and selforganizing person tracking in sensor networks. This network consists of smart camera nodes. Object observation in such networks essentially consists of three tasks. The object tracking task detects objects, associates these with trajectories and so provides consistent object-ids. The pattern recognition analyses the collected trajectories. Based on this analysis, typical characteristics about the object movement can be estimated. This prior knowledge can be used as input to the configuration task of the sensor network. Each of these tasks is represented by software agents which provide their service to each other. We show that the collaborative solution improves the performance of the system. Performance itself is measured in a quality matrix, which is a benchmark on how an agent performs his task. Generally each task is equipped with a global quality metric $\left(\mathrm{Q}_{o t}, \mathrm{Q}_{p r}, \mathrm{Q}_{c o}\right)$. The quality measure of the object tracking agent $\left(\mathrm{Q}_{o t}\right)$ is built upon the length of the individual trajectories. The pattern recognition on persons trajectories needs a minimum trajectory length to analyze the trajectory and to predict the moving direction with a high confidence level. It is also important to include information about where an individual has come from, possible target distances, similarities of the current trajectory to existing trajectories and what other individuals have done in the same situation before. The probability that an individual reaches a possible target is calculated by a function which contains factors describing the needed information. In this case the quality metric $\left(\mathrm{Q}_{p r}\right)$ corresponds to the confidence level. The configuration agent aims at a high number of tracked objects under the constraints of the pattern recognition. This can be interpreted as the sum over the length of all trajectories as metric $\left(\mathrm{Q}_{c o}\right)$. Therefore the agent estimates the time the object is in its viewing range. Objects which can be tracked together will be handled and tracked as a group. Based on these analyses the agent generates a schedule. Objects which cannot be integrated in the schedule will be offered to neighboring cameras using the results of the pattern recognition. It is evident that the agents' goals depend on each other. This is also depicted in Fig. 1 below.

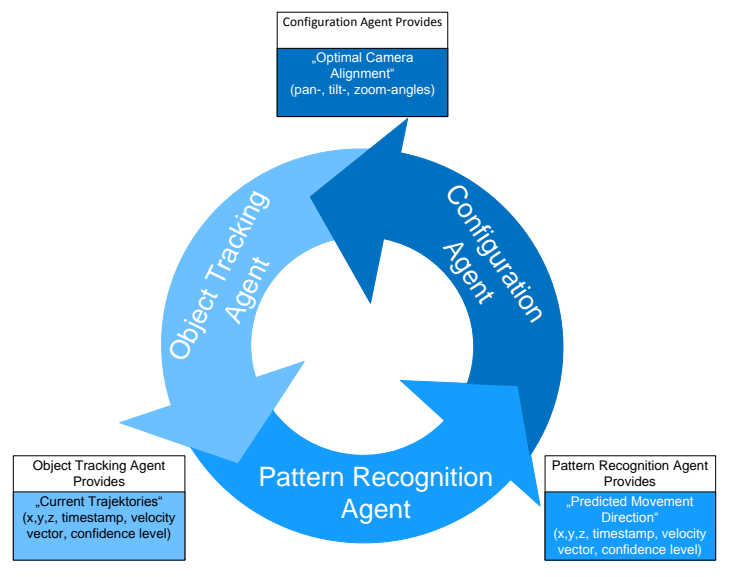

Figure 1: Circle of collaboration

A holistic measure of the success of the collaboration can be defined as the face of a kiviat diagram which axes are defined by the quality metrics of each agent. This paper shows that the collaboration will increase the amount of detected trajectory points.

\section{SYSTEM DESCRIPTION}

Intelligent surveillance systems can be classified by their degree of autonomy and capability to satisfy self-X properties like selforganization and self-configuration. The separation has been introduced by Velastin et al. (Velastin and Remagnino, 2006). They 
distinguish between three states of evolution. Systems of the first generation are classified by using analogues CCTV techniques for image distribution and storage in a single control room. Surveillance of the second generation also called semi-automatic systems use automated visual surveillance by combining computer vision and CCTV (Closed Circuit Television) systems. We want to achieve a partial contribution to systems of the third generation, fully automated wide-area surveillance systems. These systems are characterized by distribution of intelligence and also using different collaborative sensor nodes. The considered observation system within this article consists of several pan-tilt-zoom (PTZ) capable smart cameras, see Fig. 2. A smart camera simplified consists of an optical sensor and a computation unit and was first introduced by (Schneidermann, 1975). The smart camera is an automated system. The output of the optical sensor will be processed by the local computation unit, so no image data has to be transferred using communication. The network traffic can be reduced to event- and status-messages. The several observation tasks are represented by software-agents. In this work we regard the object tracking, pattern recognition and the camera alignment as agents.

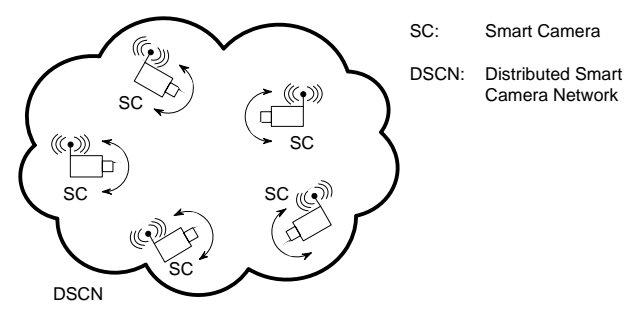

Figure 2: System overview

\section{STATE OF THE ART}

In this paper we present a holistic approach for object tracking, pattern recognition on trajectories and reconfiguration of camera networks. The separated components have been subject of numerous investigations. The design of a holistic system has been neglected several years, which was also mentioned by (Velastin and Remagnino, 2006). In order to design an automated tracking system, it is possible to access many publications which address special topics of the object tracking research field. (Everts et al., 2007) presented a system with multiple calibrated PTZ cameras which are used to track objects. The tracking and calibration results are combined, whereby the cameras can pass trackable objects to each other. Furthermore a real-time prototype system, consisting of two cameras, is introduced. The evaluation focuses on image processing techniques and shows that real-time tracking is possible with multiple PTZ cameras. (Ukita, 2005) describe a system consisting of smart-cameras so called Active Vision Agents which can track objects cooperatively. The focus is on multiple-view tracking rather than on scheduling of object tracking tasks beyond a wide area smart camera network. In (Quaritsch et al., 2007), the authors describe a method for object tracking with embedded smart cameras, i.e. the cameras have been implemented in specialized hardware. They pursued an agent-based design method. A tracking agent is responsible for the detection, identification and tracking of objects over time in a video stream of a single camera. These agents migrate through the network to follow the corresponding object. Using non-PTZ capable cameras, migration areas are defined within the image which causes a transfer of the agent to a corresponding neighboring camera if an object enters this field. The focus is on a hardware-based implementation and was evaluated on three advanced smart cameras. (Monari and Kroschel, 2010) show a task-oriented sensor selection algorithm approach for multi-camera object tracking using non-PTZ capable cameras. The algorithm aims at a highly reduced network and processor load by disabling unnecessary nodes. Our goal is to use the results of the pattern recognition on the trajectory data to align the cameras field-of-view (FoV) to optimally utilize the limited camera resources.

\section{OBJECT TRACKING}

The purpose of the object tracking agent is to gather trajectories, i.e. the locations $(X, Y, Z, t)$ of people passing the scene sampled over a sequence of images in accordance with a motion model. This agent provides the input to the pattern recognition agent (see Fig. 1). The pattern recognition agent requires preferably long trajectories to provide the configuration agent with proper prior knowledge. We scan each image with a sliding window based pedestrian detector and use the responses as evidence for the presence of people. From literature one can conclude that variants of the HOG / SVM framework perform very well under our viewing conditions (Enzweiler and Gavrila, 2009), i.e. pedestrians appear in a range of approx. 50-200 pixel vertical elongation. For pedestrian detection we thus follow (Dalal and Triggs, 2005), and classify Histograms of Oriented Gradients (HOG) with a support vector machine as either people or non-people. In order to suppress false alarms, a new target is only initialised, if the detection coincides with what is declared as foreground, i.e. if the centre pixel of the region classified as human belongs to the foreground. Foreground regions are found by applying a Gaussian Mixture Model as in (Stauffer and Grimson, 1999). For each frame the RGB colour space is transformed into HSV representation and the hue image is masked with the background region. For initialisation a target is described by the static representation of its hue histogram as observed at the moment of detection. In order to evaluate only pixels that belong to the target, we calculate this histogram by using exactly those pixel inside the bounding box indicated by the detector that belong to the foreground region. For tracking, correspondences between a target's trajectory and a candidate region of the current masked hue image are established with the candidate that best matches the target template. The matches are found using the mean shift algorithm as in (Comaniciu et al., 2003). The footprint of the bounding ellipse that best explains the target's state is appended to the trajectory. Finally, we associate a motion model to each target in terms of a linear Kalman Filter, which smoothes the trajectory into a more plausible shape and can be used for gating the search space for recovering objects after occlusions in future work. A schematic of the workflow for detection and tracking is depicted in Fig. 3. In our testing scenario, the atrium of our university, people appear mostly isolated, as depicted in Fig. 4. The yellow grid in the graphic is equally spaced with $1 \mathrm{~m}$ in world coordinates. The trajectory of the tracked person is shown as a red line (raw detection) and a green line (filtered) respectively. The green box indicates the result of the HOG detector and the ellipse the output of the mean shift method. If an object completely leaves the visible scope of observation and re-enters the scene, the tracker does not recover this object but initialises it as a new target. Each trajectory is appended with a quality measure $Q_{o t_{i}}$ which can be accounted by the further tasks. We define a flag $Q_{o t_{i}} \in[0,1]$ that indicates if the trajectory approaches the image border where the mean shift tracker might potentially fail due to partial visibility of the people.

\section{PATTERN RECOGNITION}

The analysis of the trajectories provided by the tracking agent is the task of the pattern recognition agent. Next to other ob- 


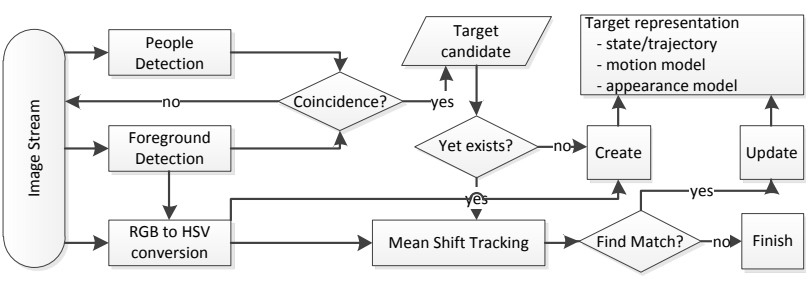

Figure 3: Workflow Detection and Tracking

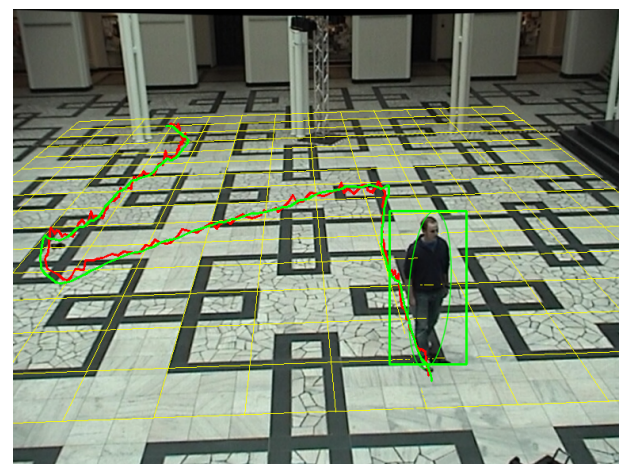

Figure 4: Tracking a single person in the atrium sequence.

jectives, which depend on the overall system goal, this analysis contains the prediction of movements to estimate future positions of objects. This prediction knowledge is demanded by the given system structure. Since we are using few smart cameras with a limited FoV, we are not able to observe the entire scene completely. There are 'dark' gaps, in which moving objects may disappear from tracking. Due to the fact that the configuration agent, which receives the results of the pattern recognition agent, aims to avoid gaps within tracks, it requires knowledge, how to adjust the cameras optimally. This knowledge is gained by the prediction objective. This objective also contains the evaluation of the predictions with the help of a quality metric $Q_{p r}$. The latter is derived by the significance and the reliability of the calculated values that indicate the most possible future position. The pattern recognition is also more efficient, if the basis of the analysis, namely the trajectories, are more complete and reliable as well. There are various possibilities to predict movements. Often and in the simplest case a future location $l_{t+\Delta t}$ is calculated by a linear relationship of the current location $l_{t}$ and velocity vector $v_{t}$ like $l_{t+\Delta t}=l_{t}+\Delta t v_{t}$. In most cases objects do not behave like this. Their movements are initiated or influenced by many factors. For instance, those factors are described by a social force model in (Helbing and Molnar, 1995). Furthermore, since we are motivated by implementing a camera tracking system, we need to use a prediction method which is able to operate at runtime. Considering those features we use an approach containing several consecutive algorithms. The first one, which is described in detail in (Feuerhake et al., 2011), creates and updates the basis for our prediction algorithm. The result of this first step is a graph structure (see Fig. 5), which is incrementally built up by extracted interesting places and a segmentation of trajectories, which clusters trajectory segments connecting the same start and end places. The resulting graph is an input for the second step. In this step the next possible destinations and the corresponding probabilities of moving objects are calculated. The second step consists of the prediction algorithm. Statements about possible paths of an object are made with the help of the graph at every time step. Each of those statements is quantified by a probability value. The calculation of such a value always refers to the current position of the object and all leaving edges from the last it has reached. First of all, statistics of all outgoing trajectories can be set up to yield probability values of a possible decision. How- ever, a decision will also depend on additional factors (see Fig. 6), e.g.: the way selection other objects have taken before (a), the distance to possible destinations (b), the similarities concerning the shape of the current to other way segments (c) as well as the already passed way (d).

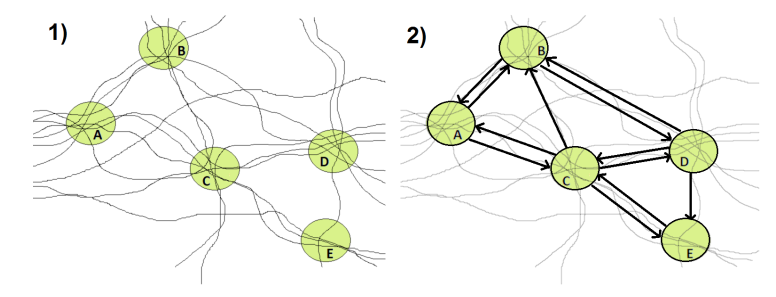

Figure 5: Using a places extraction algorithm for creating the graph as basis for the prediction algorithm

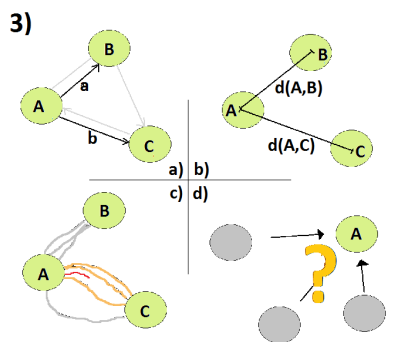

Figure 6: The probability depends on four factors: (a) preferred destination of other individuals, (b) distance to possible destinations, (c) similarities concerning shape and parameters of the current to other way segments, (d) already passed places.

We use the example in Fig. 6(c) to describe the creation of the probability values of the red object trajectory, which has left the place A. The first factor can be derived by the relative frequency the edge of the graph was used, which connects the last node $(A)$ and the possible destination node $X=A, B, C$. So let $x_{1}, . ., x_{n}$ be the usage counts of the edges leaving node $A$ and $x_{1}$ be the count of the edge connecting $A$ and $X$. Then the probability value $P_{o w}$ of destination $X$ is calculated by

$$
P_{\text {ow }}(X)=\frac{x_{1}}{\sum_{i=1}^{n} x_{i}}
$$

It does not depend on the individual's current position and remains the same until another place is entered. The next factor compares distances to possible destinations. Let $P$ be the current position of an individual. The relationship between distances $d(P,$.$) and probability P_{d}$ of node $B$ can be described by

$$
P_{d}(X)=1-\frac{d(P, X)}{\sum_{i=1}^{n} d\left(P, X_{i}\right)}
$$

For considering the shape of the way an individual has passed since the last place the Hausdorff distances between the segment bundles, which are already stored in the edges of the graph, and the current segment are calculated. Let $s_{1}, . ., s_{n}$ be the segment bundles leading accordingly to the destination nodes, $c$ the current segment and $d_{h}(.,$.$) the Hausdorff distance. Then the prob-$ ability $P_{c w}(X)$ an individual is moving to $X$ is calculated by

$$
P_{c w}(X)=1-\frac{d_{h}\left(c, s_{1}\right)}{\sum_{i=1}^{n} d_{h}\left(c, s_{i}\right)}
$$


The last factor deals with the history of visited places. The probability is described by the relative frequency of a given sequence in a subset of all sequences. Let $S_{1}, . ., S_{n}$ be the corresponding sequences ending at the given destinations and containing a given subsequence of nodes (previous node, current node). This leads to the following relationship

$$
P_{p w}(X)=\frac{S_{1}}{\sum_{i=1}^{n} S_{i}}
$$

Since the introduced factors give independent hints for the next probable destination, we combine them by summing them up. At the same time we weight them. Those weights are used to normalize the probability value and to handle different scenarios, where the relevance of each factor differs. For instance, given a scenario, it is known a priori that the distances to possible targets play a minor role, the relevance of the distance factor $P_{d}$ can be reduced by decreasing the its weight $w_{d}$. In general, if there is no a priori knowledge, the factors should be equally weighted. An auto-determination of the optimal weight setting is planned. Compared to the example shown in Fig.7(a) the overall probability $P(X)$ for the next visited place including the components described above is

$$
\begin{array}{r}
P(X)=\omega_{o w} \cdot P_{o w}(X)+\omega_{d} \cdot P_{d}(X) \\
+\omega_{c w} \cdot P_{c w}(X)+\omega_{p w} \cdot P_{p w}(X)
\end{array}
$$

with

$$
\omega_{o w}+\omega_{d}+\omega_{c w}+\omega_{p w}=1
$$

As most of the factors heavily depend on the completeness and the quality measurement $Q_{o t}$ of the tracking agent to filter out bad tracks, the results of the pattern recognition agent also depend on these features. So prediction statements of the most probable destination of an object become more significant and reliable, which means that the quality $Q_{p r}$ increases as well.
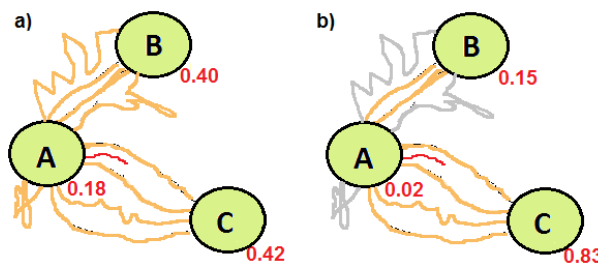

Figure 7: Example for the different results of the destination prediction of an object in the presence of bad tracks (red: current segment, orange: considered segments, grey: neglected segments)

\section{CAMERA ALIGNMENT FOR OBJECT TRACKING}

The configuration agent is responsible to align the FoV to optimally exploit the limited sensor resources. Such a scenario is depicted in Fig. 8. The moving objects (squares) are observed by smart cameras (triangles). Basically, our solution is subjected to the following constraints: We have a limited number of smart cameras. The path of individual objects through the network is unknown. The goal of the configuration agent is a reasonable tracking of individual objects and to achieve the maximum system performance with respect to the constraints of a distributed system. This leads to the question how "system performance" can be defined. The configuration agent tries to increase the amount of trajectory points $(t p)$ which are detected by the tracking agent for each object $(o b j I D)$ for each time step $(t)$. A formal description is given by equation 7 .

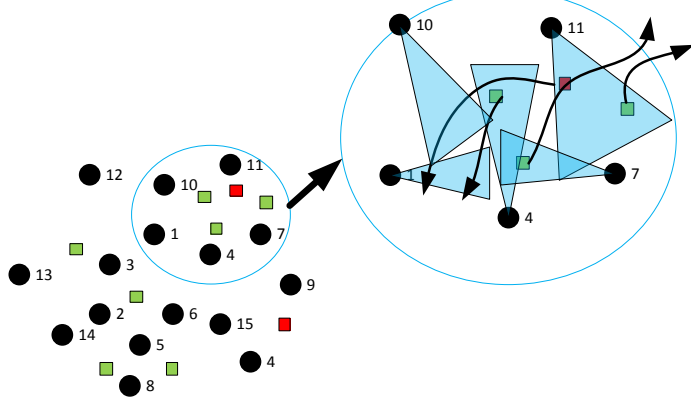

Figure 8: Coverage Agent

In order to consider the priority of an object in security scenarios $(t p)$ can be weighted with a parameter describing the priority.

$$
\begin{gathered}
\max \left\{\sum_{o b j I D} \sum_{t} \operatorname{tp}(\operatorname{objID}, t)\right\} \\
t p(\operatorname{objID}, t)= \begin{cases}1, & \text { if } \operatorname{pos}(\operatorname{obj} I D) \in F o V \\
0, & \text { otherwise }\end{cases}
\end{gathered}
$$

The tracking agent provides object positions in world-coordinates $(\mathrm{X}, \mathrm{Y}, \mathrm{Z}, \mathrm{t})$ with a unique object-ID. The approach is divided into the following steps.

1. Analyzing the time detected objects will remain in the range of work of the smart camera (SC)

2. Grouping objects which can be tracked simultaneously

3. Inserting these groups into the scheduler

4. Objects which leave the viewing range of the SC will be transfered to neighboring cameras

During the first step the time which a detected object remains in the viewing range of the smart camera is estimated. For this task the velocity of the object is predicted based on linear regression of the detected trajectory points. The observation time is the time duration an object is expected to move through the viewing range of the smart camera. This is depicted in Fig. 9(a). In the second step objects which can be tracked at the same time are grouped (Fig. 9(b)). For the calculation of the tracking time as grouped targets we introduce a heuristic. To avoid an analysis whether all objects are included in the reconfigurable FoV, the footprint of the FoV are approximated by a working-circle (WC). On the left of Fig. 9(b) a pessimistic estimation of the WC diameter is depicted. An estimation based on the in-circle of the footprint is sufficient. On the right of Fig. 9(b), object movements are shown in a spacetime diagram. For illustrative purposes, the objects are depicted to move in a two-dimensional space (x/y-plane). As long as the distance of two tracked objects is less than the diameter of the working-circle, the objects can be tracked together. This 2-tuple can be combined to a 3-tuple, see Fig. 9(c). Each of those three objects has to fit in the WC during the observation time. This is fulfilled as long as the distance between each pair of these three objects is less than or equal $\frac{3}{2 \sqrt{3}} \varnothing_{W C}$. This is a very pessimistic and restrictive policy. So it is expedient to check if the distance is $\leq \varnothing_{W C}$. A 3-tuple is constructed if any 2-tuple combination of the 3-tuple is trackable. Based on 2-tuple and 3-tuple, groups of higher order can be constructed. This is depicted in Fig. 9(d). Object 1 to 4 can be followed by one camera, if they fit into the WC during the observation time. It is evident that a k-tuple $(\mathrm{k}>2)$ is trackable if any 2-tuple combination of the k-tuple is trackable. The observation time for a k-tuple is as long as the shortest time 

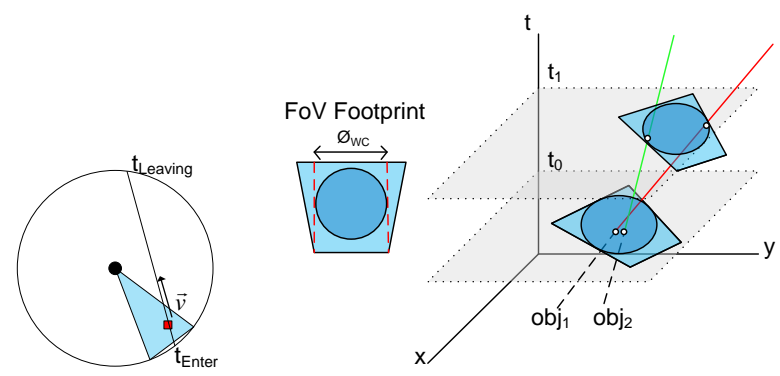

(a) Estimation (b) Estimation of the observation time for a 2of the maximum tuple of targets. The footprint of the FoV can be observation time for approximated by a working circle (WC) which is a single target (red depicted on the left. It shows a pessimistic estimasquare) based on the tion of the WC diameter, an in-circle of the footviewing range and print is a sufficient approximation. Two objects the velocity $\vec{v}$. can be tracked as a group as long as the distance is less than or equal the diameter of the WC as depicted on the right.

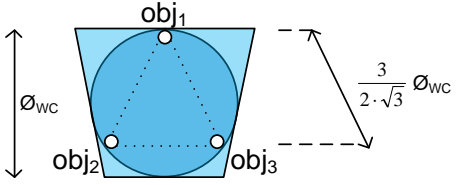

(c) A 3-tuple of objects can be tracked as a group if the distance between them is less as a group if all objects than or equal $\frac{3}{2 \sqrt{3}}$. This is a pessimistic fit into the WC. For this estimation. tions of the k-tuple including objects must be trackable as a group.

Figure 9: Calculation of k-tuple of trackable objects.

of any of the 2-tuple. Based on these predictions a scheduling graph as depicted in Fig. 10 is constructed. The configuration agent aligns the FoV according to this graph. The goal of the tracking agent is to maximize the number of detected trajectory points (Eq. 7). A simple heuristic to achieve this goal is to select the scheduling graph entry with the most objects. In Fig. 10 the configuration agent follows object 1,2,3 and 4 at time step $t_{\text {current }}$. If an object can no longer be tracked by a SC, this object will be offered to neighboring cameras. Based on the analysis of the pattern recognition agent a value is assigned to each trajectory, expressing the probability with which point of interest will be visited next. Based on this probability values a corresponding neighboring camera is selected. In Fig. 11 exemplary trajectories and three cameras are depicted. It is obvious that an object leaving the observation range of camera 1 should be offered to camera 2 because of the higher probability of re-detection.

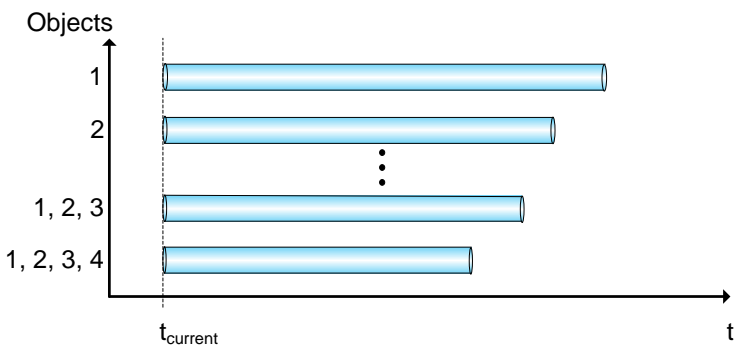

Figure 10: This scheduling graph exemplarily is showing in which time interval objects and groups of objects can be tracked.

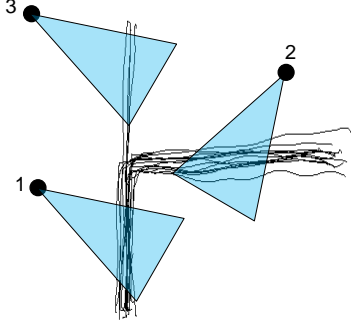

Figure 11: This figure shows exemplarily trajectories and three cameras. If an object leaves the observation range of camera 1 it will be re-detected with a higher probability in the viewing range of camera 2 than in the FoV of camera 3.

\section{RESULTS AND DISCUSSION}

For the evaluation of the described approach we recorded a videostream in the atrium of our university. The camera was positioned at a height of approximately $7 \mathrm{~m}$. In the first step the video has been analyzed by the object tracking agent. In the next step these trajectories have been analyzed by the pattern recognition agent. Each trajectory point was enriched with a list of possible destinations and a corresponding probability/confidence by the pattern recognition agent. The configuration agent was analyzed using the multi-agent simulation toolkit MASON (Luke et al., 2004). Using simulation gives us the possibility to repeat the evaluation under various conditions with the same ground truth data of the object tracking, and pattern recognition agent.

\subsection{Results of the Object Tracking Agent}

For the general case of people appearing isolated in the image, we achieve satisfying results with our tracking strategy. When two or more targets overlap in the image domain, the appearance model supports the re-association to the temporarily occluded right target (see Fig. 12) but drawbacks in the geometric accuracy of the trajectories might be encountered during occlusions. Upon visual inspection the geometric accuracy of the trajectories is not worse than half a meter, which mainly results from the rough approximation of the target position by the ellipse. We plan to overcome such drawbacks by incorporating detection based strategies rather than template tracking in future work and analyzing patterns of motion for bridging local occlusions. The strategy for data association can be improved by replacing the static appearance model with an adaptive one. Applied to a target and updated over time, such a learning step is expected to improve recognition by generalizing to a broader variety of object appearances while discriminating against other targets. The quality measure of the individual trajectories, $Q_{o t_{i}}$, is exchangeable and will be replaced with a more sophisticated measure of quality, that accounts for the particular tracker, in future work.

\subsection{Results of the Pattern Recognition Agent}

We measure the improvement of the prediction task, which results from the collaboration of the agents, by determining the increase of the correctness $\mathrm{R}$ of the prediction results. $\mathrm{R}$ represents the reliability of the predictions. The latter can be determined by comparing predicted to actual targets for each prediction. We examined five different scenarios under the condition of either using all trajectories received from the tracking agent (Case 1) or just using the trajectories with values above a certain threshold (Case 2 ). We compare the results, which consist of about 6000 predictions, in Table 7.2. While the first is showing the percentage of correct predictions using all of the prediction factors, the next 


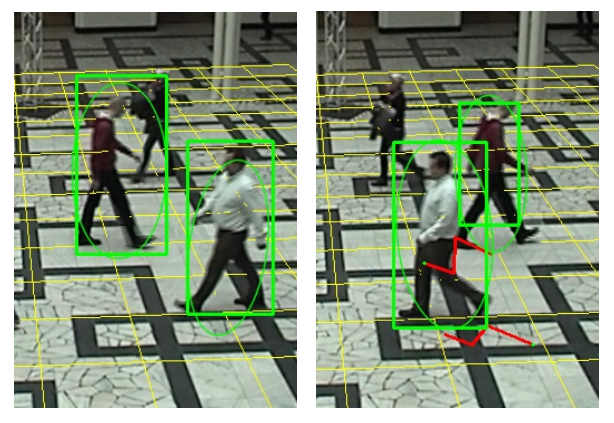

Figure 12: Tracking multiple persons with overlapping viewing ranges. Note the person in the red dress, initialized in the left image is kept tracking in the right image after partial occlusion.

\begin{tabular}{|c|c|c|}
\hline & Case 1 & Case 2 \\
\hline$R($ total $)$ & $18.02 \%$ & $43.89 \%$ \\
$R\left(P_{\text {ow }}\right)$ & $17.69 \%$ & $36.87 \%$ \\
$R\left(P_{d}\right)$ & $18.97 \%$ & $43.53 \%$ \\
$R\left(P_{c w}\right)$ & $18.72 \%$ & $39.26 \%$ \\
$R\left(P_{p w}\right)$ & $15.02 \%$ & $40.01 \%$ \\
\hline
\end{tabular}

Table 1: Percentage of correct predictions for different test cases.

four scenarios show the correctness values for each prediction ( $R=$ correctpredictions/totalpredictions) factor individually. In each of these scenarios an increase of about $20-25 \%$ is recognizable.

\subsection{Results of the Configuration Agent}

The trajectory-data (enriched by the results of the pattern recognition agent) was used as input of the multi-agent simulation toolkit. During the simulation we placed four smart cameras in the observation area, see Fig. 13. We repeated the simulation two times. During the first evaluation the configuration agents have no collaborative behavior. The neighboring cameras were not notified about possible targets in their observation area. In the next evaluation the possible destination points of the pattern recognition agent were used to notify the cameras near the predicted destination point. These cameras aligned their FOV to the expected target. During the first evaluation the cameras were capable to record 2177 trajectory points. Using collaboration between the agents increases the number of trajectory points to 2666. As described in equation 7 the number of detected trajectory points is the goal to be optimized. Using collaborative behavior increases the number of trajectory points significantly by $22 \%$.

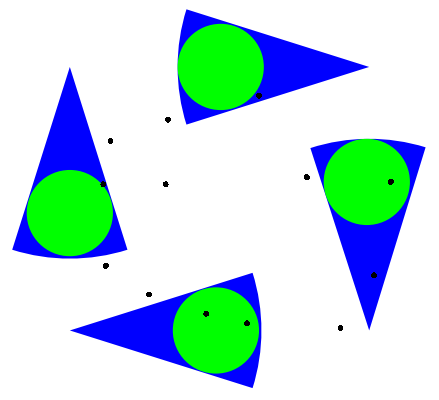

Figure 13: Screenshot of the MASON simulation toolkit. It depicts the used camera setting as it was in use during the evaluation. The black dots mark the position of the points of interest as result of the pattern recognition agent.

\section{CONCLUSIONS AND FUTURE WORK}

In this paper we presented a holistic approach for object tracking. We introduced three agent types which use collaboration to improve their individual skills. An object tracking agent is responsible to calculate trajectory points which are analyzed by a pattern recognition agent to find points of interest within the observation field. These data are then used to align the cameras field of view by the configuration agent. Neighboring cameras can notify each other about approaching objects. The evaluation shows an increasing of detected trajectory points of $22 \%$. In future work we want to integrate the software agents into smart cameras to evaluate the real-time capability.

\section{ACKNOWLEDGEMENTS}

We gratefully acknowledge the financial support by the German Research Foundation (DFG) for the project QTrajectories (DFG grant IDs: HA 5480/2-1, HE 1822/24-1, SE 645/7-1).

\section{REFERENCES}

Comaniciu, D., Ramesh, V. and Meer, P., 2003. Kernel-based object tracking. Pattern Analysis and Machine Intelligence, IEEE Transactions on 25(5), pp. $564-577$.

Dalal, N. and Triggs, B., 2005. Histograms of oriented gradients for human detection. In: Computer Vision and Pattern Recognition, 2005. CVPR 2005. IEEE Computer Society Conference on, Vol. 1, pp. $886-893$ vol. 1.

Enzweiler, M. and Gavrila, D., 2009. Monocular pedestrian detection: Survey and experiments. Pattern Analysis and Machine Intelligence, IEEE Transactions on 31(12), pp. 2179-2195.

Everts, I., Sebe, N. and Jones, G., 2007. Cooperative object tracking with multiple ptz cameras. In: Image Analysis and Processing, 2007. ICIAP 2007. 14th Int. Conf. on, pp. $323-330$.

Feuerhake, U., Kuntzsch, C. and Sester, M., 2011. Finding interesting places and characteristic patterns in spatiotemporal trajectories. In: 8th LBS Symposium 2011, pp. 4-21.

Helbing, D. and Molnar, P., 1995. Social force model for pedestrian dynamics. Physical review E 51(5), pp. 4282.

Luke, S., Cioffi-revilla, C., Panait, L. and Sullivan, K., 2004. Mason: A new multi-agent simulation toolkit. In: University of Michigan.

Monari, E. and Kroschel, K., 2010. Task-oriented object tracking in large distributed camera networks. In: Advanced Video and Signal Based Surveillance (AVSS), 2010 Seventh IEEE International Conference on, pp. $40-47$.

Quaritsch, M., Kreuzthaler, M., Rinner, B., Bischof, H. and Strobl, B., 2007. Autonomous multicamera tracking on embedded smart cameras. EURASIP J. Emb. Syst. 2007, pp. 35-35.

Schneidermann, R., 1975. Smart cameras clicking with electronic functions. Electronics 48, pp. 74-81.

Stauffer, C. and Grimson, W., 1999. Adaptive background mixture models for real-time tracking. In: Computer Vision and Pattern Recognition, 1999. IEEE Computer Society Conference on., Vol. 2, pp. 2 vol. (xxiii+637+663).

Ukita, N., 2005. Real-time cooperative multi-target tracking by dense communication among active vision agents. In: Intelligent Agent Technology, IEEE/WIC/ACM Int. Conf., pp. 664 - 671.

Velastin, S. and Remagnino, P., 2006. Intelligent Distributed Video Surveillance Systems (Professional Applications of Computing). Institution of Engineering and Technology. 